\title{
SYNTHESIS AND ANTIOXIDANT ACTIVITY OF \\ SOME CHALCONES CONTAINING \\ $N$-ARYLACETAMIDE GROUP
}

\author{
Cong Tien Nguyen ${ }^{\mathrm{a}^{*}}$, Na Pham-Thien Vo ${ }^{\mathrm{a}}$, Lien Thi-Hong \\ Nguyen $^{\mathrm{a}}$, Khue Nguyen-Thuy To ${ }^{\mathrm{a}}$, Kiet Van Doan ${ }^{\mathrm{b}}$ and Hieu \\ Quang $\operatorname{Tran}^{\mathrm{c}}$
}

${ }^{a}$ Faculty of Chemistry, Ho Chi Minh City University of Education, 280 An Duong Vuoong Street, 700000, Ho Chi Minh City, Vietnam

${ }^{b}$ Faculty of Biology and Chemistry, Tay Bac University, Quyet Tam Ward, Son La City, 34000, Son La Provine, Vietnam

${ }^{c}$ Department of Food Technology, Saigon Technology University, 180 Cao Lo Street, 700000, Ho Chi Minh City, Vietnam

\begin{abstract}
E)-3-(4-chlorophenyl)-1-(4-hydroxyphenyl)prop-2-en-1-one (3a) or (E)-3-(4-methoxyphenyl)-1-(4-hydroxyphenyl)prop-2-en-1-one

(3b) were prepared from reaction of 4-hydroxyacetophenone (1) and 4-chlorobenzaldehyde (2a) or 4-methoxybenzaldehyde (2b), respectively. The reaction of $\mathbf{3 a}$ or $\mathbf{3 b}$ and various $\mathrm{N}$-aryl-2-chloroacetamides (4a-d) afforded eight new $\mathrm{N}$-aryl-2-(4-(3-(4substituted phenyl)acryloyl)phenoxy)acetamide compounds (5a-h). The structures of the compounds were confirmed by IR, ${ }^{1} \mathrm{H}-\mathrm{NMR},{ }^{13} \mathrm{C}-\mathrm{NMR}$ and $\mathrm{HR}-\mathrm{MS}$ spectral data. Antioxidant activity performed by DPPH radical scavenging method showed that $N$-aryl-2-(4-(3-(4-substituted phenyl)acryloyl)phenoxy)acetamide compounds (5a-h) at a concentration of $10.0 \mu \mathrm{g} / \mathrm{mL}$ possess antioxidant activity in equivalent to that of ascorbic acid at a concentration of $6.0-8.0 \mu \mathrm{g} / \mathrm{mL}$ while the antioxidant activity of $\mathbf{3} \mathbf{a}$ and $\mathbf{3 b}$ compounds is higher that of ascorbic acid at the same concentration.
\end{abstract}

Keywords: Acetamide, Antioxidant activity, Chalcones, 4-Chlorobenzaldehyde, 4-Hydroxyacetophenone, 4-Methoxybenzaldehyde

\footnotetext{
*Cong, Nguyen-Tien, e-mail: congnt@hcmue.edu.vn
} 


\section{Introduction}

Chalcone is a common natural compound and one of the important intermediates in the biosynthesis of flavonoids. Chalcones also are important starting materials for the synthesis of biologically important heterocycles, such as pyrazolines, ${ }^{1,2}$ isoxazolines, ${ }^{2}$ benzodiazepines, ${ }^{3}$ benzothiazepines, ${ }^{4}$ etc. Some of the substituted chalcones and their derivatives have been reported to possess some interesting biological properties, including anti-inflammatory, ${ }^{5}$ anticancer, ${ }^{5-7}$ antioxidant, ${ }^{8,9}$ antimicrobial $^{10}$ and anti-diabetic activities. ${ }^{11}$ Besides that, compounds with phenoxy- $N$-arylacetamide scaffold have demonstrated a variety of biological activities such as antimicrobial, ${ }^{12-15}$ antioxidant, ${ }^{15,16}$ anticancer, ${ }^{17,18}$ anti-inflammatory, ${ }^{15,18}$ analgesic, ${ }^{18,19}$ antipyretic, ${ }^{18,19}$ and enzyme inhibitory, ${ }^{20-25}$ HIV inhibitory ${ }^{26}$ activities.

This paper described the preparation of chalcones containing phenoxy- $N$-arylacetamide scaffold and their antimicrobial activities to look for new useful compounds for pharmaceutical chemistry.

\section{Results and Discussion}

The reaction pathway for synthesis of (E)- $N$-aryl-2-(4-(3-(4chloro/4-methoxyphenyl)acryloyl)phenoxy)acetamides (4a-h) is illustrated in Scheme 1.

The chalcones $(\mathbf{3 a}, \mathbf{b})$ were prepared by the condensation of 4'-hydroxyacetophenone (1) with 4-chlorobenzaldehyde (2a) or 4-methoxybenzaldehyde (2b), respectively under alkaline conditions as described in the literature. ${ }^{7,10}$ Physical properties and IR, ${ }^{1} \mathrm{H}-\mathrm{NMR}$ spectral data of $(\mathbf{3 a}, \mathbf{b})$ compounds are conformity with data in the literature. ${ }^{7}$ In the IR spectra of the $\mathbf{3 a}$ and $\mathbf{3 b}$ compounds, the signal of the $\mathrm{C}=\mathrm{O}$ group appeared in the low frequency near $1642 \mathrm{~cm}^{-1}$ because of the conjugation of the $\mathrm{C}=\mathrm{O}$ and $\mathrm{C}=\mathrm{C}$ bonds. Besides that, absorptions corresponding to bending vibrations appeared around $988 \mathrm{~cm}^{-1}$ in the spectra of these compounds indicated that $(\mathbf{3 a}, \mathbf{b})$ were trans-alkenes. In the ${ }^{1} \mathrm{H}-\mathrm{NMR}$ 
spectra, the coupling between $\alpha-\mathrm{H}(\delta$ 7.86) and $\beta-\mathrm{H}(\delta 8.08)$ with a spin-spin coupling constant $(J=17,0 \mathrm{~Hz})$ indicated that both of chalcones $(\mathbf{3 a}, \mathbf{b})$ exist in the $(E)$-configuration, which is in agreement with IR spectral data described above.

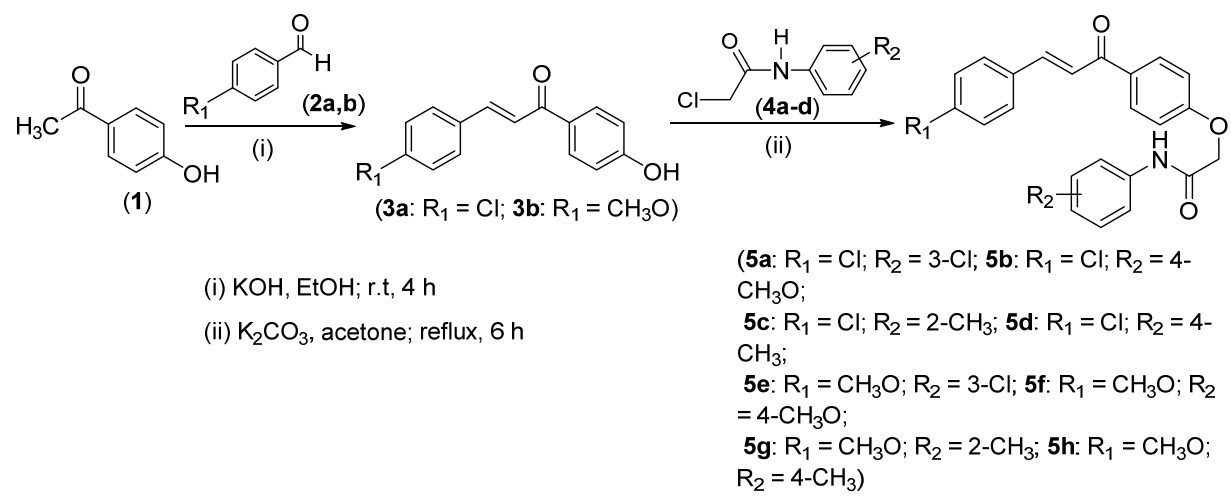

Scheme 1. The synthetic pathway.

$\mathrm{N}$-aryl-2-chloroacetamides were synthesized according to the procedure described in our previous report. ${ }^{13}$ In this procedure, $\mathrm{N}$-aryl-2-chloroacetamides were obtained with high yield by acylation of corresponding substituted anilines by using chloroacetyl chloride as an acylation agent and acetic acid as a solvent.

Stirring mixture of a definite 4-hydroxychalcone (3a or $\mathbf{3 b}$ ) and a definite $N$-aryl-2-chloroacetamide (4a or $4 \mathbf{b}$ or $4 \mathbf{c}$ or $4 \mathbf{d}$ ) in acetone containing potassium carbonate gave corresponding substituted chalcones (5a-h).

The reaction belongs to the Williamson reaction type to prepare ethers from phenols and halogen derivatives. Mass spectra of the (5a-h) compounds showed the molecular peaks in agreement with their molecular formula. The IR, ${ }^{1} \mathrm{H}$ NMR, and ${ }^{13} \mathrm{C}-\mathrm{NMR}$ spectra of the products are conformity with the proposed structures. In the IR spectra of the (5a-h) compounds, the stretching band at $3380-3415 \mathrm{~cm}^{-1}$ indicated the presence of the $\mathrm{NH}$ group while the strong band around $1690 \mathrm{~cm}^{-1}$ indicated the presence of an amide $\mathrm{C}=\mathrm{O}$ group. In comparison to the ${ }^{1} \mathrm{H}-\mathrm{NMR}$ spectra of 
N. T. Cong et al.

$(\mathbf{3 a}, \mathbf{b})$, the spectra of (5a-h) compound appeared some extra signals in the aromatic area. Moreover, the signal of the $\mathrm{CH}_{2}$ group as a singlet with the intensity of $2 \mathrm{H}$ at 4.73-4.88 ppm was seen easily. The ${ }^{1} \mathrm{H}-\mathrm{NMR}$ spectra also showed the presence of vinylic protons near $7.70 \mathrm{ppm}(\mathrm{H \alpha})$ and $7.80 \mathrm{ppm}$ $(\mathrm{H} \beta)$ with a coupling constant $\mathrm{J}_{\mathrm{ab}} \approx 15.0 \mathrm{~Hz}$ referred to trans conformation. In the ${ }^{13} \mathrm{C}$-NMR spectra of $(\mathbf{5} \mathbf{a}-\mathbf{h})$ compounds, the aliphatic carbon of the acetamide group showed a signal near $67.5 \mathrm{ppm}$.

Antioxidant capacity of chalcones (3a,b) and their acetamide derivatives (5a-h) was evaluated by 1,1-diphenyl-2-picrylhydrazyl (DPPH) radical scavenging method. ${ }^{27}$ This method is based on the spectrophotometric measurement of DPPH radical concentration changes resulting from the reaction of DPPH radical with an antioxidant. Ascorbic acid was used as a reference standard compound, and the experiment was done in triplicate. Table 1 below shows the absorbance (A) of the solution containing DPPH $(2.85 \mathrm{~mL})$ and ascorbic acid at different concentrations and inactivated DPPH percent (\% I) caused by these solutions.

A standard curve was prepared using the different concentrations of DPPH radical. The following equation of calibration curve was used to calculate the level of DPPH radical in the reaction system: $y=0.5137 \mathrm{x}+$ 6.499. The DPPH radical scavenging capacities were calculated from the obtained calibration curve determined by linear regression $\left(\mathrm{R}^{2}=0.9954\right)$.

Table 1. Free radical scavenging activities of the Ascorbic acid.

\begin{tabular}{cccccc}
\hline $\begin{array}{c}\text { Ascorbic } \\
\text { acid } \\
(\mu \mathrm{g} / \mathrm{mL})\end{array}$ & 2.0 & 4.0 & 6.0 & 8.0 & 10.0 \\
\hline $\mathrm{A}_{1}$ & 0.939 & 0.787 & 0.681 & 0.578 & 0.455 \\
$\mathrm{~A}_{2}$ & 0.933 & 0.798 & 0.683 & 0.553 & 0.544 \\
$\mathrm{~A}_{3}$ & 0.912 & 0.800 & 0.680 & 0.566 & 0.434 \\
& 0.928 & 0.795 & 0.681 & 0.566 & 0.478 \\
$\mathrm{~A}_{\text {average }}$ & \pm 0.16 & \pm 0.01 & \pm 0.00 & \pm 0.01 & \pm 0.06 \\
$\mathrm{I} \%$ & 15.64 & 27.73 & 38.06 & 48.58 & 56.58 \\
\hline
\end{tabular}


The radical scavenging activity of chalcones $(\mathbf{3 a}, \mathbf{b})$ and their acetamide derivatives (5a-h) was expressed as inactivated DPPH percent (I \%). These values were determined from the standard curve, and the results were shown in Table 2.

Table 2. The radical scavenging activities of (3a,b) and (5a-h) compounds.

\begin{tabular}{cccc}
\hline Compounds & I $(\%)$ Mean \pm SD & Compounds & I (\%) Mean \pm SD \\
\hline $\mathbf{3 a}$ & $61.22 \pm 4.32$ & $\mathbf{3 b}$ & $63.37 \pm 3.53$ \\
$\mathbf{5 a}$ & $45.21 \pm 2.67$ & $\mathbf{5 e}$ & $37.46 \pm 3.22$ \\
$\mathbf{5 b}$ & $35.26 \pm 4.21$ & $\mathbf{5 f}$ & $35.36 \pm 2.11$ \\
$\mathbf{5 c}$ & $36.82 \pm 3.16$ & $\mathbf{5 g}$ & $43.69 \pm 3.18$ \\
$\mathbf{5 d}$ & $29.58 \pm 3.22$ & $\mathbf{5 h}$ & $31.69 \pm 1.16$ \\
\hline
\end{tabular}

The results indicated that the antioxidant activity of the chalcone compounds containing acetamide group (5a-h) at a concentration of $10.0 \mu \mathrm{g} / \mathrm{mL}$ was equivalent to that of ascorbic acid at a level of about 6.0 to $8.0 \mu \mathrm{g} / \mathrm{mL}$. However, the original chalcones with the free hydroxyl group (without acetamide group) possessed higher antioxidant activity, exceeding the antioxidant activity of ascorbic acid at a concentration of $10.0 \mu \mathrm{g} / \mathrm{mL}$. The reason may be that the free hydroxyl group stabilizes free radicals and thus increases the antioxidant capacity of these compounds. There was no significant difference in the antioxidant activity of $\mathbf{3 a}$, and it's derivatives in comparison to that of $\mathbf{3 b}$ and it's derivatives.

\section{Experimental}

Melting points were determined on a Gallenkamp apparatus and were uncorrected. Infrared (IR) spectra were recorded on a Shimadzu FTIR-8400S spectrophotometer. Nuclear magnetic resonance (NMR) spectra were recorded on a Bruker Avance III spectrometer $(500 \mathrm{MHz}$ for ${ }^{1} \mathrm{H}$ NMR and $125 \mathrm{MHz}$ for ${ }^{13} \mathrm{C}$ NMR) using residual solvent DMSO- $d_{6}$ 
signals as internal references. HR-MS experiments were performed using an Agilent Q-TOF 6500.

Synthesis of (E)-3-aryl-1-(4-hydroxyphenyl)prop-2-en-1-one (3)

To solution of $4.08 \mathrm{~g}(0.03 \mathrm{~mol})$ 4-hydroxyacetophenone (1) in $60 \mathrm{~mL}$ methanol, $30 \mathrm{~mL}$ solution of potassium hydroxide $50 \%$ was slowly added while stirring for 15 minutes. To this solution, $0.03 \mathrm{~mol}$ 4-chlorobenzaldehyde (2a) or 4-methoxybenzaldehyde (2b) was added dropwise with continuous stirring for 30 minutes. The stirring was continued for another $24 \mathrm{~h}$ at room temperature, the mixture then was poured in ice-cold water further acidified with dilute $\mathrm{HCl}$. The solid obtained was filtered, recrystallized from ethanol to afford 3a (74 \%) or $\mathbf{3 b}$ (68\%) as yellow solid, respectively.

(E)-3-(4-chlorophenyl)-1-(4-hydroxyphenyl)prop-2-en-1-one (3a): m.p. 188,6 ${ }^{\circ} \mathrm{C}$ (Lit. ${ }^{7}: 188-190{ }^{\circ} \mathrm{C}$ ); IR (v, $\left.\mathrm{cm}^{-1}, \mathrm{KBr}\right): 3062,3010$ (broad, $\left.\mathrm{OH}, \mathrm{Csp}{ }^{2}-\mathrm{H}\right), 1641(\mathrm{C}=\mathrm{O}), 1582(\mathrm{C}=\mathrm{C}) ;{ }^{1} \mathrm{H}$ NMR (500 MHz, DMSO- $\left.d_{6}\right)$ : $\delta 6.92(2 \mathrm{H}, d, J=8.5, \operatorname{ArH}), 7.50(2 \mathrm{H}, d, J=8.5, \mathrm{ArH}), 7.67(1 \mathrm{H}, d$, $J=15.0, \alpha-\mathrm{H}), 7.90(2 \mathrm{H}, d, J=8.5, \mathrm{ArH}), 7.93(1 \mathrm{H}, d, J=15.0, \beta-\mathrm{H}), 8.09$ $(2 \mathrm{H}, d, J=8.5, \mathrm{ArH}), 10.45(1 \mathrm{H}, s, \mathrm{OH})$.

(E)-3-(4-methoxyphenyl)-1-(4-hydroxyphenyl)prop-2-en-1-one (3b): m.p. $185,7{ }^{\circ} \mathrm{C}$ (Lit. ${ }^{7}:$ 186-187 ${ }^{\circ} \mathrm{C}$ ); IR (v, cm $\left.{ }^{-1}, \mathrm{KBr}\right): 3113$ (broad, OH), $1643(\mathrm{C}=\mathrm{O}), 1595,1555(\mathrm{C}=\mathrm{C}) ;{ }^{1} \mathrm{H}$ NMR (500 MHz, DMSO- $\left.d_{6}\right): \delta 3.81$ $\left(3 \mathrm{H}, s, \mathrm{CH}_{3} \mathrm{O}\right), 6.92(2 \mathrm{H}, d, J=8.0, \mathrm{ArH}), 7.01(2 \mathrm{H}, d, J=8.5, \mathrm{ArH}), 7.68$ $(1 \mathrm{H}, d, J=15.0, \alpha-\mathrm{H}), 7.78(1 \mathrm{H}, d, J=15.0, \beta-\mathrm{H}), 7.82(2 \mathrm{H}, d, J=8.0$, $\operatorname{ArH}), 8.07(2 \mathrm{H}, d, J=8.5, \mathrm{ArH}), 10.39(1 \mathrm{H}, s, \mathrm{OH})$.

Synthesis of N-aryl-2-chloroacetamide compounds (4a-d)

2-Chloroacetyl chloride $6.74 \mathrm{~g}(\sim 0.055 \mathrm{~mol})$ was added dropwise to a solution of an appropriate aromatic amine $(0.05 \mathrm{~mol})$ in $20 \mathrm{~mL}$ glacial 
Synthesis and antioxidant activity of some chalcones containing N-arylacetamide group 7

acetic acid while cooling in ice-bath. After stirring in ice-bath for $30 \mathrm{~min}$ and then stirred for $1 \mathrm{~h}$ in room temperature, the reaction mixture was poured into $100 \mathrm{~mL}$ cold water containing $4.1 \mathrm{~g}(0.05 \mathrm{~mol})$ sodium acetate. The precipitate was filtered, then washed with cold water and recrystallized from ethanol.

2-chloro- $N$-(3-chlorophenyl)acetamide (4a): white crystalline solid (8.83 g, 86.6\%); mp: $106.2{ }^{\circ} \mathrm{C}$ (Lit. ${ }^{26}: 106-108{ }^{\circ} \mathrm{C}$ );

2-chloro- $N$-(4-methoxyphenyl)acetamide (4b): brown crystalline solid (8.76 g, 87.8\%); mp: $117.8{ }^{\circ} \mathrm{C}$ (Lit. $\left.{ }^{26}: 118-120{ }^{\circ} \mathrm{C}\right)$;

2-chloro- $N$-o-tolylacetamide (4c): pale brown crystalline solid (8.32 g, 90.7\%); mp: $103.6{ }^{\circ} \mathrm{C}$ ( Lit. $^{26}$ : $\left.104-106{ }^{\circ} \mathrm{C}\right)$;

2-chloro- $N$-p-tolylacetamide (4d): white crystalline solid $(8.25 \mathrm{~g}$, 89.9\%); mp: $174.5^{\circ} \mathrm{C}$ (Lit. $\left.{ }^{26}: 174-176^{\circ} \mathrm{C}\right)$.

Synthesis of (E)-2-(4-(3-arylacryloyl)phenoxy)-N-arylacetamide (5a-h)

Solution of an appropriate $N$-aryl 2-chloroacetamide (0.01 mole) (4a or $4 \mathbf{b}$ or $4 \mathbf{c}$ or $4 \mathbf{d})$ in acetone $(10 \mathrm{~mL})$ was added dropwise to stirred solution of a definite chalcone (3a or $3 \mathbf{b})(0.01 \mathrm{~mol})$ and potassium carbonate $(0.012 \mathrm{~mol})$ in dry acetone $(15 \mathrm{~mL})$. The reaction mixture was refluxed with stirring for 6 hours and then cooled to room temperature. After pouring in ice-cold water, the separated solid was filtered and recrystallized from ethanol.

(E)-2-(4-(3-(4-chlorophenyl)acryloyl)phenoxy)-N-(3-

chlorophenyl)acetamide (5a): white needle crystals, yield $61 \%$; m.p. 192-193 ${ }^{\circ} \mathrm{C}$, IR $\left(v, \mathrm{~cm}^{-1}, \mathrm{KBr}\right): 3402(\mathrm{~N}-\mathrm{H}), 3076\left(\mathrm{Csp}^{2}-\mathrm{H}\right), 2916$ $\left(\mathrm{Csp}{ }^{3}-\mathrm{H}\right), 1676$ and $1653(\mathrm{C}=\mathrm{O}), 1595$ and $1541(\mathrm{C}=\mathrm{C}) ;{ }^{1} \mathrm{H}$ NMR $\left(500 \mathrm{MHz}, \mathrm{DMSO}-d_{6}\right): \delta 4.88\left(2 \mathrm{H}, s, \mathrm{CH}_{2}\right), 7.17(3 \mathrm{H}, m, \mathrm{ArH}), 7.38$ $\left(1 \mathrm{H}, d d, J_{1}=J_{2}=8.0, \mathrm{ArH}\right), 7.55(3 \mathrm{H}, m, \mathrm{ArH}), 7.71(1 \mathrm{H}, d, J=16.0, \alpha-\mathrm{H})$, 
$7.85(1 \mathrm{H}, d, J=2.0, \mathrm{ArH}), 7.96(3 \mathrm{H}, m, \mathrm{ArH}, \beta-\mathrm{H}), 8.20(2 \mathrm{H}, d, J=8.5$, $\mathrm{ArH}), 10.35(1 \mathrm{H}, s, \mathrm{NH}) ;{ }^{13} \mathrm{C}$ NMR (125 MHz, DMSO- $\left.d_{6}\right): \delta 67.5,115.2$, 118.6, 119.6, 123.3, 124.0, 129.4, 131.0, 131.4, 133.5, 134.3, 135.4, 140.2, 142.3, 162.2, 166.9, 187.8; HR-MS calcd for $\mathrm{C}_{23} \mathrm{H}_{17} \mathrm{Cl}_{2} \mathrm{NNaO}_{3}, 448.0483$; found, $448.0472(\mathrm{M}+\mathrm{Na})^{+}$.

(E)-2-(4-(3-(4-chlorophenyl)acryloyl)phenoxy)- $\mathrm{N}-(4-$ methoxyphenyl)acetamide (5b): white needle crystals, yield $77 \%$; m.p. 226-227 ${ }^{\circ} \mathrm{C}$, IR (v, cm $\left.{ }^{-1}, \mathrm{KBr}\right): 3383(\mathrm{~N}-\mathrm{H}), 3078\left(\mathrm{Csp}^{2}-\mathrm{H}\right), 2914$ $\left(\mathrm{Csp}{ }^{3}-\mathrm{H}\right), 1672$ and $1657(\mathrm{C}=\mathrm{O}), 1599$ and $1526(\mathrm{C}=\mathrm{C}) ;{ }^{1} \mathrm{H}$ NMR $\left(500 \mathrm{MHz}, \mathrm{DMSO}-d_{6}\right): \delta 3.84\left(3 \mathrm{H}, s, \mathrm{CH}_{3} \mathrm{O}\right), 4.73\left(2 \mathrm{H}, s, \mathrm{CH}_{2}\right), 6.93$ $(2 \mathrm{H}, d, J=9.0, \mathrm{ArH}), 7.13(2 \mathrm{H}, d, J=8.5, \mathrm{ArH}), 7.43(2 \mathrm{H}, d, J=8.0, \mathrm{ArH})$, $7.51(2 \mathrm{H}, d, J=8.0, \mathrm{ArH}), 7.54(1 \mathrm{H}, d, J=15.0, \alpha-\mathrm{H}), 7.61(2 \mathrm{H}, d, J=8.5$, $\operatorname{ArH}), 7.80(1 \mathrm{H}, d, J=15.0, \beta-\mathrm{H}), 8.11(3 \mathrm{H}, d, J=8.5$, ArH and $\mathrm{NH}) ;{ }^{13} \mathrm{C}$ NMR (125 MHz, DMSO- $\left.d_{6}\right): \delta 55.5,67.5,114.3,114.7,122.0,122.1$, 129.3, 129.6, 131.1, 132.5, 136.5, 143.1, 157.0, 160.6, 188.3; HR-MS calcd for $\mathrm{C}_{24} \mathrm{H}_{20} \mathrm{ClNNaO}_{4}, 444.0979$; found, $444.1031(\mathrm{M}+\mathrm{Na})^{+}$.

(E)-2-(4-(3-(4-chlorophenyl)acryloyl)phenoxy)- $\mathrm{N}-(2-$

methylphenyl)acetamide (5c): white needle crystals, yield $78 \%$; m.p. 189-190 ${ }^{\circ} \mathrm{C}$, IR (v, cm $\left.{ }^{-1}, \mathrm{KBr}\right): 3414(\mathrm{~N}-\mathrm{H}), 3053\left(\mathrm{Csp}^{2}-\mathrm{H}\right), 2913$ $\left(\mathrm{Csp}{ }^{3}-\mathrm{H}\right), 1680$ and $1653(\mathrm{C}=\mathrm{O}), 1593$ and $1541(\mathrm{C}=\mathrm{C}) ;{ }^{1} \mathrm{H}$ NMR $\left(500 \mathrm{MHz}, \mathrm{DMSO}-d_{6}\right): \delta 3.74\left(3 \mathrm{H}, s, \mathrm{CH}_{3}\right), 4.83\left(2 \mathrm{H}, s, \mathrm{CH}_{2}\right), 6.91(2 \mathrm{H}, d$, $J=8.5, \operatorname{ArH}), 7.17(2 \mathrm{H}, d, J=9.0, \mathrm{ArH}), 7.54(4 \mathrm{H}, m, \mathrm{ArH}), 7.71(1 \mathrm{H}, d$, $J=15.0, \alpha-\mathrm{H}), 7.93(2 \mathrm{H}, d, J=8.5, \operatorname{ArH}), 7.99(1 \mathrm{H}, d, J=15.0, \beta-\mathrm{H}), 8.20$ $(2 \mathrm{H}, d, J=9.0, \mathrm{ArH}), 10.03(1 \mathrm{H}, s, \mathrm{NH}) ;{ }^{13} \mathrm{C} \mathrm{NMR}\left(125 \mathrm{MHz}, \mathrm{DMSO}-d_{6}\right)$ : $\delta 55.7,67.6,114.4,115.2,121.8,123.3,129.4,131.0,131.3,131.4,131.9$, $134.3,135.4,142.3,156.1,162.3,166.0,187.8$; HR-MS calcd for $\mathrm{C}_{24} \mathrm{H}_{20} \mathrm{ClNNaO}_{3}, 428.1029$; found, $428.1054(\mathrm{M}+\mathrm{Na})^{+}$. 
(E)-2-(4-(3-(4-chlorophenyl)acryloyl)phenoxy)- $\mathrm{N}-(4-$

methylphenyl)acetamide (5d): white needle crystals, yield $89 \%$; m.p. 234-235 ${ }^{\circ} \mathrm{C}$, IR (v, cm $\left.{ }^{-1}, \mathrm{KBr}\right): 3393(\mathrm{~N}-\mathrm{H}), 3040\left(\mathrm{Csp}{ }^{2}-\mathrm{H}\right), 2918$ $\left(\mathrm{Csp}{ }^{3}-\mathrm{H}\right), 1684$ and $1649(\mathrm{C}=\mathrm{O}), 1593$ and $1530(\mathrm{C}=\mathrm{C}) ;{ }^{1} \mathrm{H}$ NMR (500 MHz, DMSO-d $)$ : $\delta 2.27\left(3 \mathrm{H}, s, \mathrm{CH}_{3}\right), 4.84\left(2 \mathrm{H}, s, \mathrm{CH}_{2}\right), 7.14(2 \mathrm{H}, d$, $J=8.5, \mathrm{ArH}), 7.17(2 \mathrm{H}, d, J=8.5, \mathrm{ArH}), 7.53(4 \mathrm{H}, m, \mathrm{ArH}), 7.71(1 \mathrm{H}, d$, $J=15.0, \alpha-\mathrm{H}), 7.93(2 \mathrm{H}, d, J=8.5, \operatorname{ArH}), 7.98(1 \mathrm{H}, d, J=15.0, \beta-\mathrm{H}), 8.20$ $(2 \mathrm{H}, d, J=8.5, \mathrm{ArH}), 10.08(1 \mathrm{H}, s, \mathrm{NH}) ;{ }^{13} \mathrm{C}$ NMR $\left(125 \mathrm{MHz}, \mathrm{DMSO}-d_{6}\right)$ : $\delta 20.9,67.6,115.2,120.2,123.3,129.4,129.6,131.0,131.3,131.4,133.2$, $134.3,135.4,136.3,142.3,162.3,166.2,187.8$; HR-MS calcd for $\mathrm{C}_{24} \mathrm{H}_{21} \mathrm{ClNO}_{3}, 406.1210$; found, $406.1240(\mathrm{M}+\mathrm{H})^{+}$.

(E)-2-(4-(3-(4-methoxyphenyl)acryloyl)phenoxy)- $\mathrm{N}-(3-$

chlorophenyl)acetamide (5e): white needle crystals, yield $66 \%$; m.p. 162-163 ${ }^{\circ} \mathrm{C}$, IR (v, cm $\left.{ }^{-1}, \mathrm{KBr}\right): 3404(\mathrm{~N}-\mathrm{H}), 3076\left(\mathrm{Csp}^{2}-\mathrm{H}\right), 2930$ $\left(\mathrm{Csp}{ }^{3}-\mathrm{H}\right), 1684$ and $1653(\mathrm{C}=\mathrm{O}), 1593$ and $1508(\mathrm{C}=\mathrm{C}) ;{ }^{1} \mathrm{H}$ NMR (500 MHz, DMSO-d $)$ : $\delta 3.83\left(3 \mathrm{H}, s, \mathrm{CH}_{3}\right), 4.88\left(2 \mathrm{H}, s, \mathrm{CH}_{2}\right), 7.02(2 \mathrm{H}, d$, $J=8.5, \mathrm{ArH}), 7.16(3 \mathrm{H}, m, \mathrm{ArH}), 7.38\left(1 \mathrm{H}, d d, J_{1}=J_{2}=8.0, \mathrm{ArH}\right), 7.56$ $(1 \mathrm{H}, d, J=8.0, \mathrm{ArH}), 7.70(1 \mathrm{H}, d, J=15.0, \alpha-\mathrm{H}), 7.82(1 \mathrm{H}, d, J=15.0$, $\beta-\mathrm{H}), 7.84(3 \mathrm{H}, m, \mathrm{ArH}), 8.18(2 \mathrm{H}, d, J=8.5, \mathrm{ArH}), 10.35(1 \mathrm{H}, s, \mathrm{NH}) ;{ }^{13} \mathrm{C}$ NMR (125 MHz, DMSO-d $\left.d_{6}\right): \delta 55.8,67.5,114.9,115.2,118.6,119.7$, $120.0,124.0,127.9,131.0,131.1,131.2,131.8,133.6,140.3,143.7,161.7$, 162.0, 167.0, 187.8; HR-MS calcd for $\mathrm{C}_{24} \mathrm{H}_{20} \mathrm{ClNNaO}_{4}, 444.0979$; found, $444.1032(\mathrm{M}+\mathrm{Na})^{+}$.

(E)-2-(4-(3-(4-methoxyphenyl)acryloyl)phenoxy)-N-(4-

methoxyphenyl)acetamide (5f): white needle crystals, yield $65 \%$; m.p. 173-174 ${ }^{\circ} \mathrm{C}$, IR (v, cm $\left.{ }^{-1}, \mathrm{KBr}\right): 3402$ and $3226(\mathrm{~N}-\mathrm{H}), 3072\left(\mathrm{Csp}^{2}-\mathrm{H}\right)$, $2914\left(\mathrm{Csp}^{3}-\mathrm{H}\right), 1684$ and $1661(\mathrm{C}=\mathrm{O}), 1597$ and $1528(\mathrm{C}=\mathrm{C}) ;{ }^{1} \mathrm{H}$ NMR $\left(500 \mathrm{MHz}, \mathrm{DMSO}-d_{6}\right): \delta 3.74\left(3 \mathrm{H}, s, \mathrm{OCH}_{3}\right), 3.83\left(3 \mathrm{H}, s, \mathrm{OCH}_{3}\right), 4.82(2 \mathrm{H}$, 
$\left.s, \mathrm{CH}_{2}\right), 6.92(2 \mathrm{H}, d, J=9.0, \mathrm{ArH}), 7.02(2 \mathrm{H}, d, J=8.5, \mathrm{ArH}), 7.16(2 \mathrm{H}, d$, $J=9.0, \mathrm{ArH}), 7.56(2 \mathrm{H}, d, J=9.0, \mathrm{ArH}), 7.70(1 \mathrm{H}, d, J=15.0, \alpha-\mathrm{H}), 7.82$ $(1 \mathrm{H}, d, J=15.0, \beta-\mathrm{H}), 7.85(2 \mathrm{H}, d, J=8.5, \mathrm{ArH}), 8.18(2 \mathrm{H}, d, J=8.5$, $\mathrm{ArH}), 10.03(1 \mathrm{H}, s, \mathrm{NH}) ;{ }^{13} \mathrm{C}$ NMR $\left(125 \mathrm{MHz}, \mathrm{DMSO}-d_{6}\right): \delta 55.7,55.8$, 67.6, 114.4, 114.9, 115.2, 120.0, 121.9, 127.9, 131.1, 131.2, 131.7, 131.9, 143.7, 156.1, 161.7, 162.1, 166.0, 187.8; HR-MS calcd for $\mathrm{C}_{25} \mathrm{H}_{23} \mathrm{NNaO}_{5}$, 440.1474; found, $440.1463(\mathrm{M}+\mathrm{Na})^{+}$.

\section{(E)-2-(4-(3-(4-methoxyphenyl)acryloyl)phenoxy)- $\mathrm{N}-(2-$}

methylphenyl)acetamide (5g): white needle crystals, yield $71 \%$; m.p. $180-181{ }^{\circ} \mathrm{C}$, IR (v, cm $\left.{ }^{-1}, \mathrm{KBr}\right): 3408$ and $3277(\mathrm{~N}-\mathrm{H}), 3067\left(\mathrm{Csp}^{2}-\mathrm{H}\right)$, $2918\left(\mathrm{Csp}^{3}-\mathrm{H}\right), 1668(\mathrm{C}=\mathrm{O}), 1591$ and $1533(\mathrm{C}=\mathrm{C})$; ${ }^{1} \mathrm{H}$ NMR $(500 \mathrm{MHz}$, DMSO- $\left.d_{6}\right): \delta 2.27\left(3 \mathrm{H}, s, \mathrm{CH}_{3}\right), 3.89\left(3 \mathrm{H}, s, \mathrm{OCH}_{3}\right), 4.77\left(2 \mathrm{H}, s, \mathrm{CH}_{2}\right), 6.97$ $(2 \mathrm{H}, d, J=8.5, \mathrm{ArH}), 7.12(3 \mathrm{H}, m, \mathrm{ArH}), 7.24(2 \mathrm{H}, m, \mathrm{ArH}), 7.44(1 \mathrm{H}, d$, $J=15.0, \alpha-\mathrm{H}), 7.63(2 \mathrm{H}, d, J=9.0, \mathrm{ArH}), 7.82(1 \mathrm{H}, d, J=15.0, \beta-\mathrm{H}), 7.97$ $(1 \mathrm{H}, d, J=8.5, \mathrm{ArH}), 8.10(2 \mathrm{H}, d, J=9.0, \mathrm{ArH}), 8.20(1 \mathrm{H}, s, \mathrm{NH}) ;{ }^{13} \mathrm{C}$ NMR (125 MHz, DMSO- $\left.d_{6}\right): \delta 17.5,55.4,67.7,114.5,114.6,119.3,122.5$, 125.6, 127.0, 127.7, 128.7, 130.2, 130.6, 131.0, 133.0, 134.6, 144.5, 136.3, 160.3, 161.7, 165.4, 188.6; HR-MS calcd for $\mathrm{C}_{25} \mathrm{H}_{23} \mathrm{NNaO}_{4}, 424.1525$; found, $424.1563(\mathrm{M}+\mathrm{Na})^{+}$.

(E)-2-(4-(3-(4-methoxyphenyl)acryloyl)phenoxy)- $\mathrm{N}-(4-$

methylphenyl)acetamide (5h): white needle crystals, yield $70 \%$; m.p. 187-188 ${ }^{\circ} \mathrm{C}$, IR $\left(v, \mathrm{~cm}^{-1}, \mathrm{KBr}\right): 3401(\mathrm{~N}-\mathrm{H}), 3001\left(\mathrm{Csp}^{2}-\mathrm{H}\right), 2918$ $\left(\mathrm{Csp}{ }^{3}-\mathrm{H}\right), 1682$ and $1645(\mathrm{C}=\mathrm{O}), 1586$ and $1530(\mathrm{C}=\mathrm{C}) ;{ }^{1} \mathrm{H}$ NMR (500 MHz, DMSO-d $)$ : $\delta 2.27\left(3 \mathrm{H}, s, \mathrm{CH}_{3}\right), 3.83\left(3 \mathrm{H}, s, \mathrm{OCH}_{3}\right), 4.83(2 \mathrm{H}$, $\left.s, \mathrm{CH}_{2}\right), 7.02(2 \mathrm{H}, d, J=8.5, \mathrm{ArH}), 7.14(2 \mathrm{H}, d, J=8.0, \mathrm{ArH}), 7.15(2 \mathrm{H}, d$, $J=8.5, \operatorname{ArH}), 7.53(2 \mathrm{H}, d, J=8.0, \mathrm{ArH}), 7.70(1 \mathrm{H}, d, J=15.0, \alpha-\mathrm{H}), 7.82$ $(1 \mathrm{H}, d, J=15.0, \beta-\mathrm{H}), 7.85(2 \mathrm{H}, d, J=8.5, \mathrm{ArH}), 8.18(2 \mathrm{H}, d, J=9.0$, $\mathrm{ArH}), 10.08(1 \mathrm{H}, s, \mathrm{NH}) ;{ }^{13} \mathrm{C}$ NMR $\left(125 \mathrm{MHz}, \mathrm{DMSO}-d_{6}\right): \delta 20.9,55.9$, 

67.6, 114.9, 115.1, 120.0, 120.2, 127.9, 129.6, 131.1, 131.2, 131.7, 133.2, $136.3,143.7,161.7,162.1,166.3,187.8$; HR-MS calcd for $\mathrm{C}_{25} \mathrm{H}_{24} \mathrm{NO}_{4}$, 402.1705; found, $402.1705(\mathrm{M}+\mathrm{H})^{+}$.

\section{Antioxidant activity}

The antioxidant activity of chalcones compounds was determined by DPPH radical scavenging. ${ }^{27}$ Briefly, $1.0 \mathrm{~mL}$ of ascorbic acid solution at concentrations of: $2.0 ; 4.0 ; 6.0 ; 8.0 ; 10.0 \mu \mathrm{g} / \mathrm{mL}$ was added to the test tubes. Then add $2.85 \mathrm{~mL}$ of DPPH solution at $40 \mu \mathrm{g} / \mathrm{mL}$ of concentration and shake well. Leave at room temperature in the dark for 30 minutes. Absorbance was measured at $517 \mathrm{~nm}$. The results were shown in Table 1 . Then $10.0 \mu \mathrm{g} / \mathrm{mL}$ of concentration of different synthesized compounds was also prepared in methanol. All sample solutions, $1.0 \mathrm{~mL}$ of sample, was added $2.85 \mathrm{~mL}$ DPPH solution at $40 \mu \mathrm{g} / \mathrm{mL}$ of concentration. The test tubes were kept for $30 \mathrm{~min}$ in the dark to complete the reaction. The absorbance of each test tube was recorded at $517 \mathrm{~nm}$ on the UV-VIS spectrophotometer against methanol as a blank. The DPPH free radical scavenging activity (I\%) was calculated using the following formula: I \% $=\frac{A_{x+d}-A_{n m m a l e}}{A_{s t d}} \cdot 100$ where: $\mathrm{A}_{\text {std }}$ : absorbance of the DPPH standard sample;

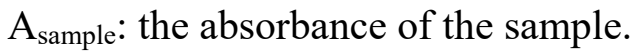

The results were shown in Table 2.

\section{Conclusions}

(E)-3-(4-chlorophenyl)-1-(4-hydroxyphenyl)prop-2-en-1-one, (E)-3-(4-methoxyphenyl)-1-(4-hydroxyphenyl)prop-2-en-1-one and eight new their $N$-aryl-2-(4-(3-(4-substituted phenyl)acryloyl)phenoxy)acetamide derivatives were synthesized. The structure of the compounds was confirmed by IR, ${ }^{1} \mathrm{H}-\mathrm{NMR},{ }^{13} \mathrm{C}-\mathrm{NMR}$, and HR-MS spectral data. The antioxidant activity of $\mathrm{N}$-aryl-2-(4-(3-(4-substituted phenyl)acryloyl)phenoxy)acetamide compounds is lower while the 


antioxidant activity of both (E)-3-(4-chlorophenyl)-1-(4-

hydroxyphenyl)prop-2-en-1-one and (E)-3-(4-methoxyphenyl)-1-(4hydroxyphenyl)prop-2-en-1-one is higher in comparison to that of ascorbic acid at the same concentration.

\section{References}

1. Nagihan, B.; Bedia, K. K.; Salih, G.; Feyza, A. Synthesis and anticonvulsant activity of some 2-pyrazolines derived from chalcones. Arab. J. Chem. 2017, 10(2), S2073-S2081.

2. Voskienè, A.; Mickevičius, V. Cyclization of chalcones to isoxazole and pyrazole derivatives. Chem. Heterocycl. Compd. 2009, 45, 1485-1488.

3. Kamal, M. E. Application of chalcone in synthesis of new heterocycles containing 1,5-benzodiazepine derivatives. Am. J. Org. Chem. 2014, 4(1), 14-19.

4. Cong, T. N.; Ha, M. B.; Duyen, H. M. L. Synthesis of 2-(2-(4-phenyl-2,3dihydrobenzo[b][1,4]thiazepin-2-yl)phenoxy)- $N$-( $p$-tolyl)acetamide. Acta Chemica Iasi, 2018, 26(1), 13-20.

5. Won, S. J.; Liu, C. T.; Tsao, L. T.; Weng, J. R.; Ko, H. H.; Wang, J. P.; Lin, C. $\mathrm{N}$. Synthetic chalcones as potential anti-inflammatory and cancer chemopreventive agents. Eur. J. Med. Chem. 2005, 40(1), 103-112.

6. Suvitha, S.; Abdelwahab, S. I.; Al-Mamary, M. A.; Mohan, S. Synthesis of chalcones with anticancer activities. Molecules, 2012, 17, 6179-6195.

7. Chairil, A.; Yogo, D. P.; Sabirin, M.; Winarto, H.; Eti, N. S.; Nendrowati. Synthesis of chalcone derivatives and their in vitro anticancer test against breast (T47D) and colon (WiDr) cancer cell line. Indones. J. Chem. 2018, 18(1), 102-107.

8. Venkatachalam, H.; Yogendra, N.; Jayashree, B. S. Evaluation of the antioxidant activity of novel synthetic chalcones and flavonols. Int. J. Chem. Eng. Appl. 2012, 3(3), 216-219.

9. Jiabing, W.; Lili, H.; Chanchan, C.; Ge, L.; Jingwen, X.; Mengya, S.; Qian, C.; Wulan L.; Wenfei, H.; Peihong, Q.; Jianzhang, W. Design, synthesis and biological evaluation of chalcone analogues with novel dual antioxidant mechanisms as potential anti-ischemic stroke agents. Acta Pharm. Sin. B, 2019, 9(2), 335-350.

10. Ngaini, Z.; Haris-Fadzillah, S. M.; Hussain, H.; Kamaruddin, K. Synthesis and antimicrobial studies of (E)-3-(4-alkoxyphenyl)-1-(2-hydoxyphenyl)prop-2-en1-one, (E)-3-(4-alkoxyphenyl)-1-(4-hydoxyphenyl)prop-2-en-1-one and their analogues. World J. Chem. 2009, 4(1), 9-14. 
Synthesis and antioxidant activity of some chalcones containing $\mathrm{N}$-arylacetamide group $\mathbf{1 3}$

11. Chi-Ting, H.; Tusty-Jiuan, H.; El-Shazly, M.; Da-Wei, C.; Yi-Hong, T.; ChiaoTing, Y.; Shou-Fang, W.; Yang-Chang, W.; Fang-Rong, C. Synthesis of chalcone derivatives as potential anti-diabetic agents. Bioorg. Med. Chem. Lett. 2012, 22(12), 3912-3915.

12. Berest, G. G.; Voskoboynik, O. Y.; Kovalenko, S. I.; Antypenko, O. M.; Nosulenko, I. S.; Katsev, A. M.; Olena, S. S. Synthesis and biological activity of novel $N$-cycloalkyl-(cycloalkylaryl)-2-[(3- $R$-2-oxo-2H-[1,2,4]triazino[2,3c]quinazoline-6-yl)thio]acetamides. Eur. J. Med. Chem. 2011, 46, 6066-6074.

13. Cong, N. T.; Duc, T. T. C.; Ha, B. M.; Dat, N. D. Synthesis and antibacterial activity of some derivatives of 2-methylbenzimidazole containing 1,3,4oxadiazole or 1,2,4-triazole heterocycle. J. Chem. 2016, 1507049.

14. John, D. W.; Matthew, C. T.; Venugopal, R. N.; Carson, B.; Nicholas, O. B.; Ming, D.; McCarthy, C. T.; Daniel, A.; Norton, P. P.; Terry, L. B.; Donald, T. M. Synthesis and structure-activity relationships of novel phenoxyacetamide inhibitors of the Pseudomonas aeruginosa type III secretion system (T3SS). Bioorg. Med. Chem. 2015, 23(5), 1027-1043.

15. Vikas, G. R.; Kiran, G.; Vinayak, K. D. Synthesis, characterization and study of some $N$-substituted aryl-2-(\{4-[(substituted aryl carbamoyl)methyl]-5(pyridin-4-yl)-4H-1,2,4-triazol-3-yl $\}$ sulfanyl)acetamide. Iran. J. Pharm. Sci. 2014, 10(2), 21-34.

16. Autore, G.; Anna, C.; Stefania, M.; Barbara, N.; Chiara, P.; Aldo, P.; Ada, P.; Maria, S. S.; Giuseppina, T.; Carmela, S. Acetamide derivatives with antioxidant activity and potential anti-inflammatory activity. Molecules 2010, 15, 2028-2038.

17. Priyanka, R.; Dilipkumar, P.; Rahul, R. H.; Syed, R. H. Anticancer, antiinflammatory, and analgesic activities of synthesized 2-(substituted phenoxy) acetamide derivatives. J. Chem. 2014, 386473.

18. Adimule, V.; Medapa, S.; Kumar, S.; Rao, P. K. Design, Synthesis, characterization and anticancer properties of novel 2-chloro- $N$-(aryl substituted)acetamide derivatives of 5-[2-(4-methoxyphenyl) pyridin-3-yl]-1, 3, 4-oxadiazole-2-thiol. Int. J. Drug Dev. \& Res. 2014, 6(4), 188-195.

19. Priyanka, R.; Dilip, K. P.; Rahul, R. H.; Syed, R. H. Synthesis, characterization and pharmacological evaluation of substituted phenoxy acetamide derivatives. Hem. Ind. 2015, 69(4), 405-415.

20. Süreyya, Ö.; Pinar, V.; Tülay, Ç.; Dogu, N. J. Synthesis and evaluation of $\mathrm{N}$-substituted indole-3-carboxamide derivatives as inhibitors of lipid peroxidation and superoxide anion formation. Enzyme Inhib. Med. Chem. 2008, 23(3), 334-340. 
21. Nulgumnalli, M. R.; Aitha, J.; Alapati, V. R.; Subrahmanyam, C.V.S. Synthesis, pharmacological evaluation and docking studies of $N$-(benzo[ $d]$ thiazol-2-yl)-2-(piperazin-1-yl)acetamide analogs as COX-2 inhibitors. Bioorg. Med. Chem. Lett. 2012, 22(2), 820-823.

22. Kilic-Kurt, Z.; Bakar, F.; Ölgen, S. N-(3-Substituted-benzylidene-2oxoindolin-5-yl)acetamide derivatives as Src kinase inhibitors: Synthesis, biological evaluation and molecular docking studies. Austin J. Anal. Pharm. Chem. 2015, 2(5), 1052-1057.

23. Ashish, K. S.; Jyoti, P.; Nimisha, J.; Pradeep, K. S. QSAR studies of 2-phenoxyacetamide analogues, a novel class of potent and selective monoamine oxidase inhibitors. Curr. Res. Pharm. Sci. 2017, 7(4), 109-116.

24. Fu-Chun, Z.; Yan, W.; Xiao-Jie, S. Design and development of a novel chalcone derivative as an anticholinesterase inhibitor for possible treatment of dementia. Med. Sci. Monit. 2017, 23, 3311-3317.

25. Benjamin, N. A.; David, S.; Yuguang, Z.; James, S.; Luca, V.; Reinis, R. R.; Fiona, J.; Georgie, L.; Sarah, F.; Amy, M.; Svend, K.; Magda, B.; Jones, E. Y.; Paul, V. F. Discovery of 2-phenoxyacetamides as inhibitors of the Wnt-depalmitoleating enzyme NOTUM from an X-ray fragment screen. Med. Chem. Commun. 2019, 10, 1361-1369.

26. Murugesan, S.; Swastika, G.; Giovanni, M. Synthesis, evaluation and molecular modeling studies of some novel tetrahydroisoquinoline derivatives targeted at the HIV-1 reverse transcriptase. Pharm. Lett. 2011, 3(6), 317-332.

27. Meng, G. E.; Tian, Y. C.; Yang, Y.; Shi, S. Evaluation of DPPH free radical scavenging activity of various extracts of Ligularia fischeri in vitro: a case study of Shaanxi region. J. Indian J. Pharm. Sci. 2016, 78(4), 436-442. 